\title{
Determination of Cardiac Ejection Fraction by Electrical Impedance Tomography - Numerical Experiments and Viability Analysis
}

\author{
Franciane C. Peters, Luis Paulo S. Barra, and Rodrigo Weber dos Santos \\ Universidade Federal de Juiz de Fora, Juiz de Fora, Minas Gerais, Brasil \\ franciane.peters@engenharia.ufjf.br, luis.barra@ufjf.edu.br, \\ rodrigo.weber@ufjf.edu.br
}

\begin{abstract}
Cardiac ejection fraction is a clinically relevant parameter that is highly correlated to the functional status of the heart. Today the non-invasive methods and technology that measure cardiac ejection fraction, such as MRI, CT and echocardiography do not offer a continuous way of monitoring this important parameter. In this work, we numerically evaluate a new method for the continuous estimation of cardiac ejection fraction based on Electrical Impedance Tomography. The proposed technique assumes the existence of recent Magnetic Resonance (MR) images of the heart to reduce the search space of the inverse problem. Simulations were performed on two-dimensional cardiac MRI images with electric potentials numerically obtained by the solution of the Poisson equation via the Boundary Element Method. Different protocols for current injection were evaluated. Preliminary results are presented and the potentialities and limitations of the proposed technique are discussed.
\end{abstract}

\section{Introduction}

Cardiac ejection fraction indicates the measure of the blood fraction that is pumped from each ventricle in each step of the heart cycle. The ejection fraction of both left ventricle (EFLV) and right ventricle (EFRV) can be determined. But the clinical use of EFLV is more common and it is frequently called ejection fraction (EF). By definition, the ejection fraction is calculated in the following way:

$$
E F=\frac{P V}{E D V}=\frac{E D V-E S V}{E D V}
$$

where $P V$ is the volume of blood pumped, that is given by the difference between the end-diastolic volume $(E D V)$ and the end-systolic volume $(E S V)$. Cardiac ejection fraction is a relevant parameter that is highly correlated to the functional status of the heart. To determine EF, different techniques can be used, like echocardiography, cardiac magnetic resonance and computed tomography. However, because of the costs of these techniques, they can not be used for continuous monitoring. In this work, we numerically evaluate a new method for the continuous estimation of cardiac ejection fraction based on Electrical Impedance Tomography (EIT). 
EIT is a technique that reconstruct conductivity distribution images inside a conductor domain based on protocols of current injection and potential measurement on the boundary of the domain. This technique has a large utilization on geophysics, monitoring of industrial activities [1] and on biomedical engineering [2 34 4. In this last area, the EIT is considered as a viable technique for monitoring long periods, since it is not based on ionizing radiation. The EIT spacial resolution is not as high as the traditional imaging methods. Nevertheless, its portability, low cost and time resolution are the main advantages of the technique.

The aim of the present work is to present a study on the viability of EIT to the continuous monitoring of cardiac ejection fraction. Previous work have shown preliminary results on the same topic [567]. In this work, we extend previous results and give new contribution in many aspects: 1) We develop a more realistic $2 \mathrm{D}$ model that includes the lungs. Because of their low conductivity, these regions behave as a barrier to the electrical currents and poses new challenges to the problem. 2) The results presented in this work were obtained using the Levenberg-Marquardt method [8] for the solution of the inverse problem associated to EIT. Previous work have adopted different methods, such as the Powell's method 9], Genetic Algorithms 1011 and FAIPA (Feasible Arc Interior Point Algorithm) [12] 3) Different protocols for current injection are evaluated for the estimation of cardiac ejection fraction.

Preliminary results are presented and the potentialities and limitations of the proposed technique are discussed. The results suggest the proposed technique is a promising diagnostic tool that offers continuous and non-invasive estimation of cardiac ejection fraction.

\section{Methods}

\subsection{D Models Based on Magnetic Resonance Images}

From magnetic resonance (MR) images, the regions of interest, in this case the two ventricles were manually segmented in two different phase: end of the systole and the end of diastole. Each curve of the segmentation was parameterized by a spline, with a minimum number of points. The left ventricle (LV) spline has 7 control points and the right one (RV) 8 points. The external boundary of the thorax and the boundaries of the lungs were also segmented. For simplicity, these curves are assumed constant during the heart cycle. Figure 1 shows a segmentation example.

The goal of our method is to recover the shape of the internal cavi ties of the heart, presently considered in two-dimensions, from electric potential measurements. Therefore, with two coordinates for each spline control point the methods would need to estimate a total of $30\left((7+8)^{*} 2\right)$ parameters. To reduce the number of parameters to be estimated the following strategy was adopted.

During MRI segmentation we have used the same number of control points for the splines in both systole and diastole phase. This allows us to restrict the search space forcing that each control point $i$ belongs to a line that connects the position 


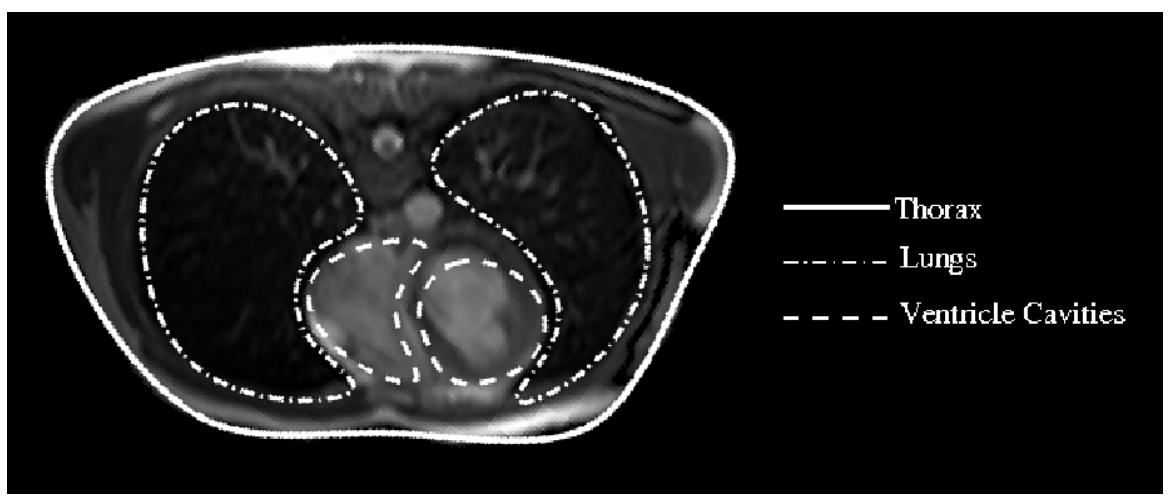

Fig. 1. Manual segmentation of an MR image. The boundary of the lungs is represented in blue and the boundary of the ventricle cavities in red.

at systole and diastole. Thus, a linear interpolation is used, parameterized by a scalar $t_{i}$, between the values of the coordinates of each control point $i$. The spline relative to the end of systole can be obtained with $t_{i}=0, \forall i$, and the one relative to the end of diastole with $t_{i}=1, \forall i$. Doing so, the method goal is to recover the shape of the ventricular cavities via the estimation of the 15 parameters $t_{i}$, with $i=1 \ldots 15$.

\subsection{Forward Problem and Governing Equations}

The proposed 2D model splits the domain in regions that represent different biological tissues, heart cavities, lungs and torso. Each tissue can be mainly electrically identified by its conductivity. Grimnes [13] presents the main factors that influence the properties of biological tissues. Although they may be classified in only four groups, epithelium, muscle, connective tissue and nervous tissue, the tissues can be divided in thirty kinds in accordance to their electrical properties 14. In addition, the value of the conductivity of each tissue depends on the frequency of the electrical current, on the temperature, on the presence of water, among other issues.

Table 1. Resistivity values of biological tissues that are found in the literature

\begin{tabular}{ccc}
\hline Tissue & Resistivity $(\Omega \mathrm{cm})$ & Reference \\
\hline \multirow{3}{*}{ Blood } & 150 & Barber and Brown [16] \\
& 150 & Yang and Patterson [17] \\
& 100 & Schwan and Kay [18] \\
\hline \multirow{2}{*}{ Heart } & 400 & Patterson and Zhang [19] \\
& 250 & Yang and Patterson [17] \\
& $400-800$ & Baysal and Eyuboglu [20] \\
\hline \multirow{2}{*}{ Lung } & $727-2363$ & Barber and Brown [16] \\
& 1400 & Patterson and Zhang [19] \\
\hline
\end{tabular}


In this work, we assume the conductivity of each tissue is taken as known, constant and isotropic. These are all simplified assumption, since biological tissues are usually heterogeneous and anisotropic. However, biological tissues are difficult to characterize, and the reported values vary substantially in the literature. Table 1 presents some resistivity values for blood, heart and lung found in the literature.

For the remaining tissues that compose the section of the thorax, that from here on we call the torso region, Bruder et al. [15] proposes a mean resistivity of $500 \Omega \mathrm{cm}$. The conductivity of the air is $10^{20} \Omega \mathrm{cm}$, but the conductivity of the lung filled of air is difficult to determine. Rush et al. 21] presents a very simplified resistivity distribution model characterized by the presence of cavities filled of blood, surrounded by homogeneous material with resistivity ten times greater. The same scheme, properly extended to include the lung regions, is used in this work. Preliminarily, the resistivity of the blood is here taken as $100 \Omega \mathrm{cm}$ and the torso to be $1000 \Omega \mathrm{cm}$. Two different values were tested for the resistivity of the lungs: $20000 \Omega \mathrm{cm}$ (Ratio of Lung to Torso resistivity (RLT) of 20) and $50000 \Omega \mathrm{cm}$ (RLT of 50).

The forward problem consists of calculating the electrical potential on the external boundary of the torso that is generated by the current injection on a pair of electrodes. Given that our 2D model has three regions with different but constant and isotropic conductivities (heart cavities, lungs and torso) the electrical potential at each point of the regions, $\phi$, must satisfy Laplaces' equation:

$$
\nabla^{2} \phi=0
$$

and the boundary conditions are

$$
\begin{array}{rc}
\sigma_{L} \nabla \phi=\sigma_{T} \nabla \phi, & \mathbf{x} \in \Gamma_{1} \\
\sigma_{B} \nabla \phi=\sigma_{T} \nabla \phi, & \mathbf{x} \in \Gamma_{2} \\
\sigma_{T} \frac{\partial \phi}{\partial \mathbf{n}}=J_{i}, & \mathbf{x} \in \Gamma_{3}^{i e} \\
\frac{\partial \phi}{\partial \mathbf{n}}=0, & \mathbf{x} \in\left(\Gamma_{3}-\Gamma_{3}^{i e}\right)
\end{array}
$$

where $\Gamma_{1}$ is the interface between the lung and torso region, $\Gamma_{2}$ is the interface between the blood and the torso region, $\Gamma_{3}$ is the external boundary of the thorax, $\Gamma_{3}^{i e}$ is the part of $\Gamma_{3}$ where the ith electrode is, $J_{i}$ is the electrical current injected on the ith electrode and $\sigma_{L}, \sigma_{B}$ and $\sigma_{T}$ are the conductivities of the lung, blood and torso, respectively.

In the present work, the forward problem is solved by the Boundary Element Method (BEM) [22]. Further details of the implementation can be found in [23].

\subsection{The Inverse Problem}

The inverse problem associated to EIT aims to recover the shape of the ventricular cavities via the estimation of the vector $\mathbf{t}$, that contains the 15 parameters $t_{i}$, with $i=1 \ldots 15$ (as described in Section 2.1). This is done via the minimization 
of an objective function that measures the distance between measured electrical potential values $\left(\bar{\phi}_{j}\right)$ taken from a pair of electrodes identified by $j$ on the external boundary and the computed ones $\left(\phi(\mathbf{t})_{j}\right)$ that depends on the heart cavity shape parameterized by $\mathbf{t}$ and is calculated as described in Section 2.2 Therefore, the goal is to find the best parameter vector $\mathbf{t}$ that minimizes Eq. 3 .

$$
F=\frac{1}{2} \sum_{j=1}^{m}\left(\phi(\mathbf{t})_{j}-\bar{\phi}_{j}\right)^{2}
$$

where $m$ is the number of measurements and depends on the current injection pattern. In this work, the "measured" electrical potential values $\left(\bar{\phi}_{j}\right)$ were also synthetically generated, i.e., also numerically obtained.

This minimization problem is solved by the method known as LevenbergMarquardt 8]. The implementation of the method and the subroutines that compute the objective function are done in Fortran77.

\subsection{Numerical Experiments and Stimulation Patterns}

For the $2 \mathrm{D}$ problem in consideration the areas of the transversal section of the cavities were assumed to be proportional to their volumes, i.e. a cylindrical approximation so that $\mathrm{EF}$ is calculate by:

$$
E F=\frac{E D A-E S A}{E D A}
$$

where $E D A$ and $E S A$ are the areas of the transversal section of the ventricle at the end of the diastole and at the end of the systole, respectively.

From MR images taken at the end of the systole and at the end of the diastole the cardiac ventricles were manually segmented and in accordance to (4) the $\mathrm{EF}$ of the left ventricle is $59.24 \%$ and the EF of the right ventricle is $29,95 \%$. After that, a cardiac disfunction was synthetically generated. The simulated disfunction consists of a modified cardiac cycle in which the end-diastolic volume is the same as in the normal cycle but the end-systolic volume is greater than the normal one. In this new cardiac cycle, the EF of the left ventricle is $33.01 \%$ and the $\mathrm{EF}$ of the right ventricle is $16.19 \%$. These are the target values to be estimated by the here proposed method.

Two patterns of electrical current injection were tested: a diametrical one, with electrodes evenly distributed along the torso boundary; and an adaptive one, with electrodes that are near the region of interest. The first yields a set of 104 measurements and the second one yields a set of 78. The arrows of the Fig. 2 present the pairs of electrodes sequentially used for current injection in each pattern.

As mentioned before in Section 2.1] we have also tested two different 2D models. Each with a different value for the resistivity of the lungs: $20000 \Omega \mathrm{cm}$ (Ratio of Lung to Torso resistivity (RLT) of 20) and $50000 \Omega \mathrm{cm}$ (RLT of 50). Finally, for each of 4 optimization problems (2 Stimulus Patterns times 2 RLT models) we have tested the optimization method with two different initial guesses. One 


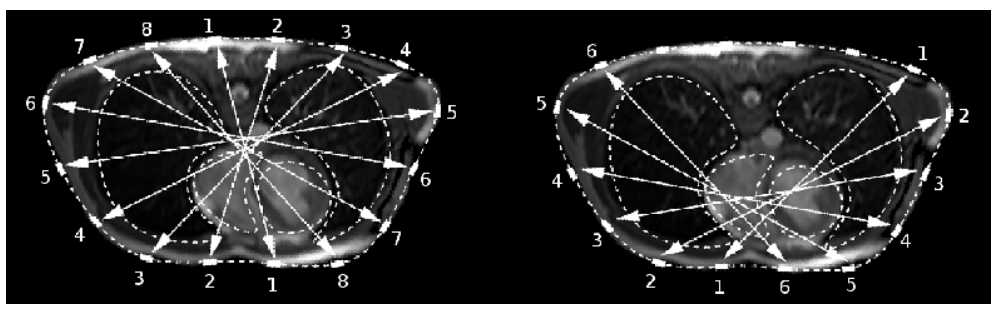

Fig. 2. Two stimulation patterns used in this work. The first one is the diametrical and the second one is the adaptive.
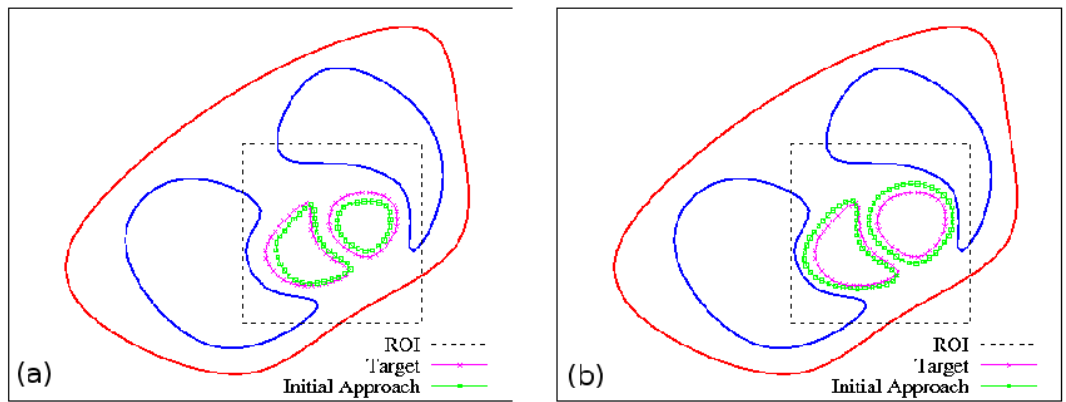

Fig. 3. A typical target (pink) and the two initial guesses (green) given to the optimization procedure: (a) $t_{i}=0, \forall i ;$ (b) $t_{i}=1, \forall i$

guess is the parameter set $\mathbf{t}$ that corresponds to the shape of the ventricles at the end of the diastole of normal heart, i.e. $t_{i}=1, \forall i$ and the other at the end of the systole for the normal tissue, i.e. $t_{i}=0, \forall i$. The initial guesses and the targets can be compared in Fig. 3. Thus, the method was executed a total of 8 times (2 Stimulus Patterns times 2 RLT models times 2 initial guesses).

\section{Results}

Table 2 presents the results of our numerical experiments that aims the EF estimation of the synthetically generated cardiac disfunction. The columns present the results for the models with different values for the resistivity of the lungs: Ratio of Lung to Torso resistivity (RLT) of 20 and RLT of 50. Each couple of rows presents the comparison of the two stimulation pattern implemented: diametrical and adaptive pattern. In addition, for each pair (stimulus pattern, RLT) results are presented for two different initial conditions. The first one corresponds to the shape of the ventricles at the end of the diastole of the normal heart, i.e. $t_{i}=1, \forall i$ and the other at the end of the systole for the normal heart, i.e. $t_{i}=0, \forall i$. The last row of the table presents the target EF values for comparison. 
Table 2. Values of the ejection fraction estimated for the synthetic cardiac disfunction for two RLT ratios and two different stimulation patterns. The last row shows the target values.

\begin{tabular}{ccccc} 
& \multicolumn{2}{c}{ RLT $=50$} & \multicolumn{2}{c}{ RLT $=20$} \\
\hline Initial & \multicolumn{2}{c}{ EF (\%) } & \multicolumn{2}{c}{ EF (\%) } \\
Guess & RV & LV & RV & LV \\
\hline \multicolumn{4}{c}{ Diametrical Pattern } \\
$t_{i}=0$ & 13.00 & 34.41 & 15.32 & 34.22 \\
$t_{i}=1$ & 16.09 & 32.21 & 15.80 & 33.04 \\
\hline \multicolumn{5}{c}{ Adaptive Pattern } \\
$t_{i}=0$ & 12.97 & 35.86 & 20.54 & 29.94 \\
$t_{i}=1$ & 18.72 & 32.84 & 20.89 & 29.40 \\
\hline Target & 16.19 & 33.01 & 16.19 & 33.01 \\
\hline
\end{tabular}

Figure 4 allows a geometrical comparison between the final results and the actual target curves. It is important to emphasize that, to make the comparison fair, the results presented in this figures are obtained with the same initial guesses, $t_{i}=1, \forall i$.

The results show that in general the error in the computed ejection fraction of the left ventricle is smaller than the one of the right. The mean absolute error of the left ventricle results is 1.64 while the right ventricle ones is 2.42. Moreover, except in one case, the diametrical pattern provides results closer to the actual values than the other pattern. The diametrical pattern provides a mean absolute error of 1.00 while the error of the adaptive pattern is 3.06. About the initial guess, both of them provided good results. But the best ones are obtained with the guess on the original diastole curve with a mean absolute error of 1.54 against an error of 2.52 for the other initial guess.

The geometrical results presented in Fig. 4. showing only the ventricular cavities, suggest that the results become worst in the case the lung resistivity is greater. This behavior is expected because greater resistivities around the region of interest tend to block the electrical current to reach this area. For instance, for the best experimented pattern, the diametrical one, the mean absolute error obtained with the greatest resistivity $(\mathrm{RLT}=50)$ is 2.2 times the error obtained with the other lung resistivity $(\mathrm{RLT}=20)$.

Using the diametrical pattern, the best result was obtained for the left ventricle and RLT of 20. The absolute error in the value of the ejection fraction is of 0.03 . It is possible to see this result in Fig. 4(b). In this case it is very difficult to see the difference between the result and the target and it is the best of the 8 tested cases. About the adaptive pattern, the best one was obtained for the left ventricle and RLT of 50. In this case, the absolute error in the value of the ejection fraction is 0.17 . 

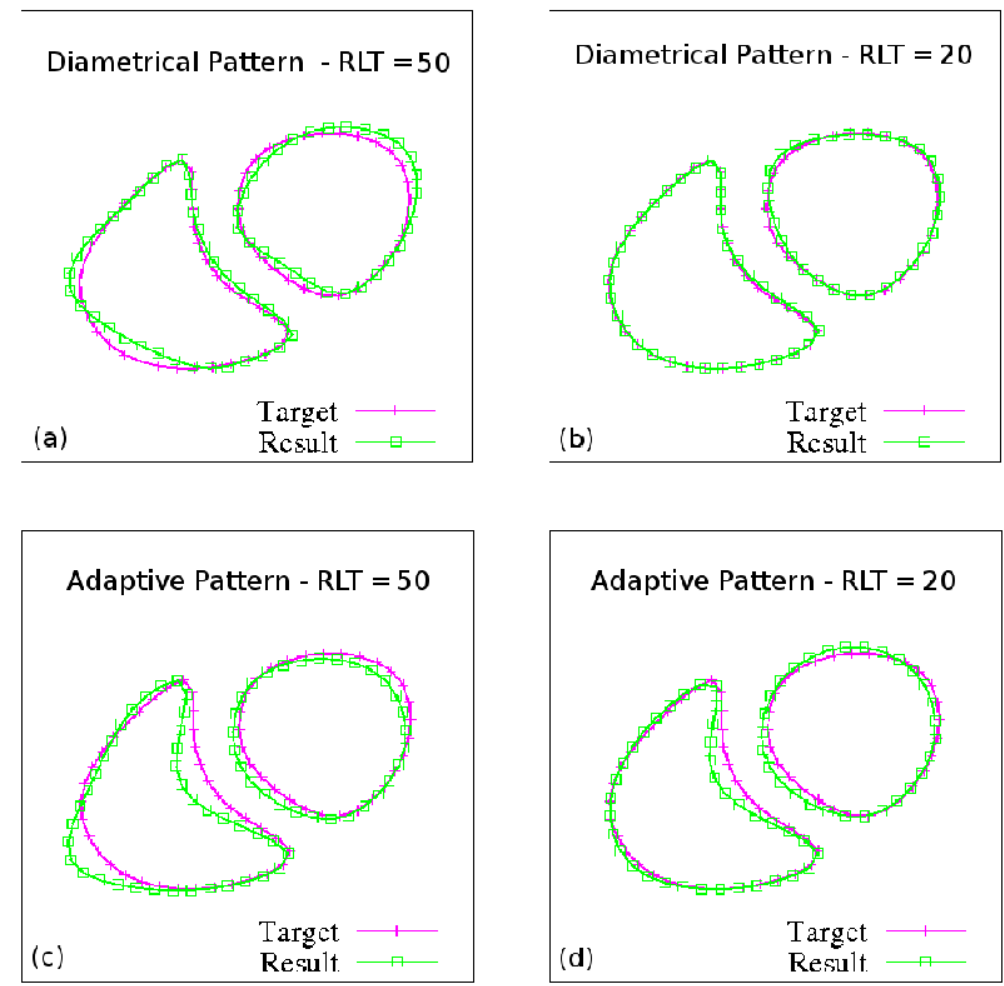

Fig. 4. Some results for the diametrical and the adaptive pattern and the target

\section{Conclusions}

The presented results suggest that the proposed methodology allows a suitable indication of the cardiac ejection fraction. We observed that the error in the ejection fraction predictions for the right ventricle are greater than those found for the left ventricle and this is in agreement with other techniques, such as with echocardiography.

Concerning the different patterns for current injection tested in this work, the errors obtained with the diametrical pattern are smaller than those using the adaptive pattern, in general. However this fact does not discard the use of the adaptive pattern, as it presents good results and spends around $19 \mathrm{~min}$. in a Pentium 4, 3.00 GHz, for a complete solution, 25\% less then the diametrical.

Comparing the results obtained with different lung resistivities we may conclude that the inverse problem becomes more difficult to be solved as the RLT increases. Therefore, the results suggest the current injection should be triggered during the expiratory phase, when the air volume and the corresponding lung resistivity are smaller. 
The preliminary results presented in this work suggest the proposed technique is a promising diagnostic tool that may offer continuous and non-invasive estimation of cardiac ejection fraction.

Acknowledgements. The first author would like to thank CAPES and Mestrado em Modelagem Computacional (UFJF) for the Master's scholarship.

\section{References}

1. Kim, M., Kim, K., Kim, S.: Phase boundary estimation in two-phase flows with electrical impedance tomography. Int. Comm. Heat Transfer 31(8), 1105-1114 (2004)

2. Polydorides, N., Lionheart, W., McCann, H.: Krylov subspace iterative techniques: On the brain activity with electrical impedance tomography. IEEE Transactions on Medical Imaging 21(6), 596-603 (2002)

3. Seo, J., Kwon, O., Ammari, H., Woo, E.: A mathematical model for breast cancer lesion estimation: Electrical impedance technique using TS2000 commercial system. IEEE Transactions on Biomedical Engineering 51(11), 1898-1906 (2004)

4. Trigo, F., Lima, R., Amato, M.: Electrical impedance tomography using extended Kalman filter. IEEE Transactions on Biomedical Engineering 51(1), 72-81 (2004)

5. Barra, L.P.S., Peters, F.C., Martins, C.P., Barbosa, H.J.C.: Computational experiments in electrical impedance tomography. In: XXVII Iberian Latin American Congress on Computational Methods in Engineering, Belém, Brasil (2006)

6. Barra, L.P.S., Santos, R.W., Peters, F.C., Santos, E.P., Barbosa, H.: Parallel computational experiments in electrical impedance tomography. In: 18th Symposium on Computer Architecture and High Performance Computing, Ouro Preto, Brasil, vol. 1, pp. 7-13. Sociedade Brasileira de Computação, High Perfomance Computing in the Life Sciences (2006)

7. Barra, L.P.S., Mappa, P., Cardoso, S., Peters, F.C.: Comparison of the Computational Performance of Optimization Algorithms in the Solution of an Inverse Problem (in Portuguese). In: VIII Simpósio Mecânica Computacional - SIMMEC, Belo Horizonte, Brasil. PUC-Minas (2008)

8. Madsen, K., Nielsen, H., Tingleff, O.: Methods for non-linear least squares problems (2004)

9. Press, W., Teukolsky, S., Vetterling, W.: Flannery: Numerical Recipes in Fortran, vol. 77. Cambridge University Press, Cambridge (1986)

10. Holland, J.: Adaptation in Natural and Artificial Systems. University of Michigan Press (1978)

11. Holland, J.: How Adaptation builds Complexity. Addison-Wesley Pub. Co., Reading (1995)

12. Herskovits, J.: Feasible direction interior-point technique for nonlinear optimization. Journal of Optimization Theory and Applications 99(1), 121-146 (1998)

13. Grimnes, S.: Bioimpedance and bioelectricity basics (2008)

14. Gabriel, C.: The dielectric properties of biological tissue: I. literature survey (1996)

15. Bruder, H., Scholz, B., Abrahamfuchs, K.: The influence of inhomogeneous volume conductor models on the ECG and the MCG. Physics in Medicine and Biology 39(11), 1949-1968 (1994)

16. Barber, D.C., Brown, B.H.: Applied potential tomography. Journal of Physics EScientific Instruments 11(9), 723-733 (1984) 
17. Yang, F., Patterson, R.P.: The contribution of the lungs to thoracic impedance measurements: a simulation study based on a high resolution finite difference model. Physiological Measurement 28(7), S153-S161 (2007)

18. Schwan, H.P., Kay, C.F.: Specific resistance of body tissues. Circulation Research 4(6), 664-670 (1956)

19. Patterson, R.P., Zhang, J.: Evaluation of an EIT reconstruction algorithm using finite difference human thorax models as phantoms. Physiological Measurement 24(2), 467-475 (2003)

20. Baysal, U., Eyuboglu, B.M.: Tissue resistivity estimation in the presence of positional and geometrical uncertainties. Physics in Medicine and Biology 45(8), 23732388 (2000)

21. Rush, S., McFee, R., Abildskov, J.A.: Resistivity of body tissues at low frequencies. Circulation Research 12(1), 40-50 (1963)

22. Brebbia, C., Telles, J.C.F., Wrobel, L.C.: Boundary Elements Techniques: Theory and Applications in Engineering. Springer, Heidelberg (1984)

23. Barra, L.P.S., Peters, F.C., Santos, R.W.: Numerical Experiments for the Viability Analysis of the Determination of the Cardiac Ejection Fraction by the Electrical Impedance Tomography (in Portuguese). In: XXIX CILAMCE - Congresso Ibero Latino Americano de Métodos Computacionais em Engenharia, Maceió, Brasil. ABMEC (2008)

24. Metherall, P.: Three Dimensional Electrical Impedance Tomography of the Human Thorax. PhD thesis, University of Sheffield (1998) 